\title{
Three-tangle for high-rank mixed states
}

\author{
Shu-Juan He${ }^{1}$, Xiao-Hong Wang ${ }^{2}$, Shao-Ming Fei ${ }^{2}$, Hong-Xiang Sun ${ }^{3}$ and Qiao-Yan Wen ${ }^{1}$ \\ ${ }^{1}$ State Key Laboratory of Networking and Switching Technology, Beijing Uni- \\ versity of Post and Telecommunication, China \\ ${ }^{2}$ Department of Mathematics, Capital Normal University, Beijing, China \\ ${ }^{3}$ School of Science, Beijing University of Post and Telecommunication, China
}

\begin{abstract}
A family of rank-n $(n=5,6,7,8)$ three-qubit mixed states are constructed. The explicit expressions for the three-tangle and optimal decompositions for all these states are given. The CKW relations for these states are also discussed.
\end{abstract}

Key words: Three-tangle, Optimal decomposition

PACS number(s): 03.67.Mn, 03.65.Ud

\section{Introduction}

Quantum entangled states are important physical resource and play key roles in quantum information processing such as teleportation, superdense coding, quantum cloning, quantum cryptography [1, 2, 3]. Characterizing and quantifying entanglement of quantum states are of great importance.

To quantify entanglement some measures like concurrence [4, entanglement of formation [5, 6] are studied. Though entanglement of bipartite states have been understood well in many aspects [7], there is still no generally accepted theory for characterizing and quantifying entanglement for multipartite qubit systems, especially for mixed states. For three-qubit systems, some results have been presented [8, 9, 10, 11]. An important quantity for three-qubit entanglement is the so called residual entanglement or three-tangle [12], which is a polynomial invariant for three-qubit states, the modulus of the hyperdeterminant [13, 14].

For a pure three-qubit state $|\psi\rangle=\sum_{i, j, k=0}^{1} a_{i j k}|i j k\rangle \in \mathcal{C}^{2} \otimes \mathcal{C}^{2} \otimes \mathcal{C}^{2}$, its three-tangle is defined by

$$
\tau_{3}(|\psi\rangle)=4\left|d_{1}-2 d_{2}+4 d_{3}\right|
$$

where

$$
d_{1}=a_{000}^{2} a_{111}^{2}+a_{001}^{2} a_{110}^{2}+a_{010}^{2} a_{101}^{2}+a_{100}^{2} a_{011}^{2},
$$




$$
\begin{gathered}
d_{2}=a_{000} a_{111} a_{011} a_{100}+a_{000} a_{111} a_{101} a_{010}+a_{000} a_{111} a_{110} a_{001} \\
+a_{011} a_{100} a_{101} a_{010}+a_{011} a_{100} a_{110} a_{001}+a_{101} a_{010} a_{110} a_{001} \\
d_{3}=a_{000} a_{110} a_{101} a_{011}+a_{111} a_{001} a_{010} a_{100}
\end{gathered}
$$

For a mixed three-qubit state $\rho=\sum_{i} p_{i} \rho_{i}, 0<p_{i} \leq 1, \rho_{i}=\left|\psi_{i}\right\rangle\left\langle\psi_{i}|,| \psi_{i}\right\rangle \in \mathcal{C}^{2} \otimes \mathcal{C}^{2} \otimes \mathcal{C}^{2}$, the three-tangle is defined in terms of convex roof [15]

$$
\tau_{3}(\rho)=\min \sum_{i} p_{i} \tau_{3}\left(\rho_{i}\right)
$$

A decomposition that realizes the above minimum is called optimal. It is a challenge to find the optimal decomposition even for the simplest case of rank-2 mixed states. Nice analytical results have been obtained for some classes of three-qubit mixed states. In [16, 17] Lohmayer et al. have constructed the optimal decomposition for a family of rank-2 three-qubit states. Jung et al.[18, 19] have also provided analytical formulae of three-tangle for a class of rank-3 and rank-4 three-qubit mixed states. In [20] a numerical method has been also presented to compute the three tangle for general three-qubit states.

In this paper we analyze the optimal decomposition for some families of high rank- $n(n=$ $5,6,7,8)$ three-qubit mixed states. The analytical expressions for the three-tangles and explicit optimal decompositions for all these states are given. The CKW relations for these states are also investigated.

\section{The Three-tangle for some high-rank mixed states}

Recently, Jung et al.[19] have provided an analytic quantification of the three-tangle for a rank-4 three-qubit mixed state which is composed by GHZ-type states. In this paper, we extend the method of [19] to the high-rank mixed states.

We use the following notations [19] in studying three tangle of high rank mixed states:

$$
\begin{array}{ll}
|G H Z, 1 \pm\rangle=\frac{1}{\sqrt{2}}(|000\rangle \pm|111\rangle), & |G H Z, 2 \pm\rangle=\frac{1}{\sqrt{2}}(|110\rangle \pm|001\rangle), \\
|G H Z, 3 \pm\rangle=\frac{1}{\sqrt{2}}(|101\rangle \pm|010\rangle), & |G H Z, 4 \pm\rangle=\frac{1}{\sqrt{2}}(|011\rangle \pm|100\rangle) .
\end{array}
$$

The case of rank-5 states We first consider the following rank-5 states:

$$
\sigma(p)=p|G H Z, 1+\rangle\langle G H Z, 1+|+(1-p) \Gamma_{G H Z},
$$

where $\Gamma_{G H Z}=\frac{1}{10}|G H Z, 1-\rangle\left\langle G H Z, 1-\left|+\frac{3}{10}\right| G H Z, 2+\right\rangle\left\langle G H Z, 2+\left|+\frac{3}{10}\right| G H Z, 3+\right\rangle\langle G H Z, 3+$ $\left.\left|+\frac{3}{10}\right| G H Z, 4+\right\rangle\langle G H Z, 4+|$.

We first consider the state $\Gamma_{G H Z}$. In order to calculate the three-tangle of the state $\Gamma_{G H Z}$, we first investigate the following rank-4 state:

$$
\rho(p)=p|G H Z, 1-\rangle\langle G H Z, 1-|+(1-p) \Pi_{G H Z},
$$


where $\Pi_{G H Z}=\frac{1}{3}[|G H Z, 2+\rangle\langle G H Z, 2+|+| G H Z, 3+\rangle\langle G H Z, 3+|+| G H Z, 4+\rangle\langle G H Z, 4+|]$ which has vanishing three-tangle [19].

By straightforward calculation the three tangle of the following pure state

$\left|Z\left(p, \varphi_{1}, \varphi_{2}, \varphi_{3}\right)\right\rangle=\sqrt{p}|G H Z, 1-\rangle-\sqrt{\frac{1-p}{3}}\left(e^{i \varphi_{1}}|G H Z, 2+\rangle+e^{i \varphi_{2}}|G H Z, 3+\rangle+e^{i \varphi_{3}}|G H Z, 4+\rangle\right)$

is given by

$$
\begin{aligned}
\tau_{3}\left(\left|Z\left(p, \varphi_{1}, \varphi_{2}, \varphi_{3}\right)\right\rangle\right)= & \mid p^{2}+\frac{(1-p)^{2}}{9}\left(e^{4 i \varphi_{1}}+e^{4 i \varphi_{2}}+e^{4 i \varphi_{3}}\right)+\frac{2}{3} p(1-p)\left(e^{2 i \varphi_{1}}+e^{2 i \varphi_{2}}+e^{2 i \varphi_{3}}\right) \\
& -\frac{2(1-p)^{2}}{9}\left(e^{2 i\left(\varphi_{1}+\varphi_{2}\right)}+e^{2 i\left(\varphi_{1}+\varphi_{3}\right)}+e^{2 i\left(\varphi_{2}+\varphi_{3}\right)}\right) \mid .
\end{aligned}
$$

Note that $\tau_{3}\left(\left|Z\left(p, \varphi_{1}, \varphi_{2}, \varphi_{3}\right)\right\rangle\right)$ is zero at $\varphi_{1}=\varphi_{2}=\varphi_{3}=0$ and $p_{0}=\frac{2-\sqrt{3}}{2} \doteq 0.134$. And $\rho(p)$ can be decomposed into $\rho(p)=\frac{p}{8 p_{0}} \sum \Gamma_{i}\left(p_{0}\right)+\frac{p_{0}-p}{p_{0}} \prod_{G H Z}$ for $0 \leq p \leq p_{0}$, where

$$
\begin{array}{ll}
\Gamma_{1}\left(p_{0}\right)=\left|Z\left(p_{0}, 0,0,0\right)\right\rangle\left\langle Z\left(p_{0}, 0,0,0\right)\right|, & \Gamma_{2}\left(p_{0}\right)=\left|Z\left(p_{0}, 0,0, \pi\right)\right\rangle\left\langle Z\left(p_{0}, 0,0, \pi\right)\right|, \\
\Gamma_{3}\left(p_{0}\right)=\left|Z\left(p_{0}, 0, \pi, 0\right)\right\rangle\left\langle Z\left(p_{0}, 0, \pi, 0\right)\right|, & \Gamma_{4}\left(p_{0}\right)=\left|Z\left(p_{0}, 0, \pi, \pi\right)\right\rangle\left\langle Z\left(p_{0}, 0, \pi, \pi\right)\right|, \\
\Gamma_{5}\left(p_{0}\right)=\left|Z\left(p_{0}, \pi, 0,0\right)\right\rangle\left\langle Z\left(p_{0}, \pi, 0,0\right)\right|, & \Gamma_{6}\left(p_{0}\right)=\left|Z\left(p_{0}, \pi, 0, \pi\right)\right\rangle\left\langle Z\left(p_{0}, \pi, 0, \pi\right),\right. \\
\Gamma_{7}\left(p_{0}\right)=\left|Z\left(p_{0}, \pi, \pi, 0\right)\right\rangle\left\langle Z\left(p_{0}, \pi, \pi, 0\right)\right|, & \Gamma_{8}\left(p_{0}\right)=\left|Z\left(p_{0}, \pi, \pi, \pi\right)\right\rangle\left\langle Z\left(p_{0}, \pi, \pi, \pi\right)\right| .
\end{array}
$$

All $\Gamma_{i}\left(p_{0}\right)$ and $\prod_{G H Z}$ 's three-tangle are zero, therefore the three-tangle for the mixed state $\rho(p)$ is zero for $0 \leq p \leq p_{0}$. Thus $\Gamma_{G H Z}$ has vanishing three-tangle.

Now consider the three-qubit pure state constituted by linear combinations of $|G H Z, 1+\rangle$, $|G H Z, 1-\rangle,|G H Z, 2+\rangle,|G H Z, 3+\rangle$ and $|G H Z, 4+\rangle$ :

$$
\begin{aligned}
\left|Z\left(p, \varphi_{1}, \varphi_{2}, \varphi_{3}, \varphi_{4}\right)\right\rangle= & \sqrt{p}|G H Z, 1+\rangle-e^{i \varphi_{1}} \sqrt{\frac{1-p}{10}}|G H Z, 1-\rangle-e^{i \varphi_{2}} \sqrt{\frac{3(1-p)}{10}}|G H Z, 2+\rangle \\
& -e^{i \varphi_{3}} \sqrt{\frac{3(1-p)}{10}}|G H Z, 3+\rangle-e^{i \varphi_{4}} \sqrt{\frac{3(1-p)}{10}}|G H Z, 4+\rangle .
\end{aligned}
$$

The corresponding three-tangle is

$$
\begin{aligned}
\tau_{3}\left(\left|Z\left(p, \varphi_{1}, \varphi_{2}, \varphi_{3}, \varphi_{4}\right)\right\rangle\right)= & \mid p^{2}+\frac{(1-p)^{2}}{100} e^{4 i \varphi_{1}}+\frac{9(1-p)^{2}}{100}\left(e^{4 i \varphi_{2}}+e^{4 i \varphi_{3}}+e^{4 i \varphi_{4}}\right) \\
& -\frac{1}{5} p(1-p) e^{2 i \varphi_{1}}-\frac{3}{5} p(1-p)\left(e^{2 i \varphi_{2}}+e^{2 i \varphi_{3}}+e^{2 i \varphi_{4}}\right) \\
& +\frac{3(1-p)^{2}}{50}\left(e^{2 i\left(\varphi_{1}+\varphi_{2}\right)}+e^{2 i\left(\varphi_{1}+\varphi_{3}\right)}+e^{2 i\left(\varphi_{1}+\varphi_{4}\right)}\right) \\
& -\frac{9(1-p)^{2}}{50}\left(e^{2 i\left(\varphi_{2}+\varphi_{3}\right)}+e^{2 i\left(\varphi_{2}+\varphi_{4}\right)}+e^{2 i\left(\varphi_{3}+\varphi_{4}\right)}\right) \\
& -\frac{6}{25} \sqrt{30} \sqrt{p(1-p)^{3}} e^{i\left(\varphi_{2}+\varphi_{3}+\varphi_{4}\right)} \mid .
\end{aligned}
$$

Since the three-tangle $\tau_{3}\left(\left|Z\left(p, \varphi_{1}, \varphi_{2}, \varphi_{3}, \varphi_{4}\right)\right\rangle\right)=0$ at $p=p_{0}=0.7377$ and $\varphi_{1}=\varphi_{2}=\varphi_{3}=$ $\varphi_{4}=0$, the state $\sigma(p)$ can be expressed in terms of $\left|Z\left(p, \varphi_{1}, \varphi_{2}, \varphi_{3}, \varphi_{4}\right)\right\rangle$,

$$
\sigma(p)=\frac{1}{8} \sum \Pi_{i}(p),
$$


where

$$
\begin{array}{ll}
\Pi_{1}(p)=|Z(p, 0,0,0,0)\rangle\langle Z(p, 0,0,0,0)|, & \Pi_{2}(p)=|Z(p, 0,0, \pi, \pi)\rangle\langle Z(p, 0,0, \pi, \pi)|, \\
\Pi_{3}(p)=|Z(p, 0, \pi, 0, \pi)\rangle\langle Z(p, 0, \pi, 0, \pi)|, & \Pi_{4}(p)=|Z(p, 0, \pi, \pi, 0)\rangle\langle Z(p, 0, \pi, \pi, 0)|, \\
\Pi_{5}(p)=|Z(p, \pi, 0,0,0)\rangle\langle Z(p, \pi, 0,0,0)|, & \Pi_{6}(p)=|Z(p, \pi, 0, \pi, \pi)\rangle\langle Z(p, \pi, 0, \pi, \pi)|, \\
\Pi_{7}(p)=|Z(p, \pi, \pi, 0, \pi)\rangle\left\langle Z(p, \pi, \pi, 0, \pi)\left|, \quad \Pi_{8}(p)=\right| Z(p, \pi, \pi, \pi, 0)\right\rangle\langle Z(p, \pi, \pi, \pi, 0)| .
\end{array}
$$

When $0 \leq p \leq p_{0}$, we have the optimal decomposition of $\sigma(p)$ :

$$
\sigma(p)=\frac{p}{8 p_{0}} \sum \Pi_{i}\left(p_{0}\right)+\frac{p_{0}-p}{p_{0}} \Gamma_{G H Z},
$$

where $\Pi_{i}(p)$ are defined as (9) $, i=1,2, \cdots, 8$. Since all $\Pi_{i}\left(p_{0}\right)$ and $\Gamma_{G H Z}$ has vanishing threetangle, we have that $\tau_{3}(\sigma(p))=0$ when $0 \leq p \leq p_{0}$.

For $p>p_{0}$, the decomposition in Eqs.(8) also is a trial optimal decomposition for $\sigma(p)$. Its three-tangle is

$$
g_{I}(p)=p^{2}-2 p(1-p)-\frac{2(1-p)^{2}}{25}-\frac{6 \sqrt{30}}{25} \sqrt{p(1-p)^{3}}, \quad p>p_{0} .
$$

We need to check whether the function $g_{I}(p)$ is convex or not for $p>p_{0}$. It can be verified that the function $g_{I}(p)$ is convex for $p<p_{*}=0.9750$, but concave for $p>p_{*}$. For large $p$ let us propose a decomposition of $\sigma(p)$ as follows:

$$
\sigma(p)=\frac{1-p}{8\left(1-p_{1}\right)} \sum \Pi_{i}\left(p_{1}\right)+\frac{p-p_{1}}{1-p_{1}}|G H Z, 1+\rangle\langle G H Z, 1+|,
$$

where $p_{1} \leq p \leq 1, p_{1} \leq p_{*}, \Pi_{i}(p)$ are defined as (9), $i=1,2, \cdots, 8$.

The three tangle of (12) is given by

$$
g_{I I}(p)=\frac{p-p_{1}}{1-p_{1}}+\frac{1-p}{1-p_{1}} g_{I}\left(p_{1}\right) .
$$

Since $d^{2} g_{\Pi} / d p^{2}=0$ for all $p$, from $\partial g_{I I} / \partial p_{1}=0$ we have

$$
3 \sqrt{30} p_{1}^{\frac{1}{2}}\left(1-p_{1}\right)^{-\frac{1}{2}}-3 \sqrt{30} p_{1}^{-\frac{1}{2}}\left(1-p_{1}\right)^{\frac{1}{2}}=73,
$$

which gives rise to

$$
p_{1}=\frac{1}{2}+\frac{73 \sqrt{6409}}{12818} \doteq 0.9559
$$

Therefore the three tangle of the rank-5 state $\sigma(p)$ is given by

$$
\tau_{3}(\sigma(p))= \begin{cases}0, & 0 \leq p \leq p_{0}, \\ g_{I}(p), & p_{0} \leq p \leq p_{1} \\ g_{I I}(p), & p_{1} \leq p \leq 1,\end{cases}
$$

where $p_{0}=0.7377, p_{1}=0.9559, g_{I}(p)$ is given by (11) and $g_{I I}(p)$ by (13). And the corresponding optimal decomposition are (10), (8) and (12) respectively. In order to show that (14) is genuine 
optimal, we plot the p-dependence of the three-tangles for various $\varphi_{1}, \varphi_{2}, \varphi_{3}, \varphi_{4}$. These curves have been referred as the characteristic curves [21. As [21] indicated, the three-tangle is a convex hull of the minimum of the characterisitic curves. Fig.1 indicates that the three-tangles plotted as black solid line are the convex characteristic curves, which implies that (14) is really optimal.

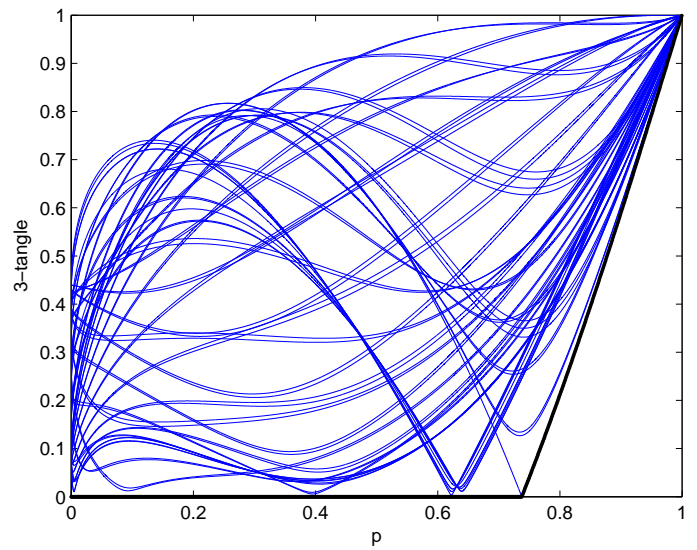

Figure 1: The $p$ dependence of various $\varphi_{1}, \varphi_{2}, \varphi_{3}, \varphi_{4}$. We have chosen $\varphi_{1}, \varphi_{2}, \varphi_{3}, \varphi_{4}$ from 0 to $2 \pi$ as interval 0.3 .

The case of rank- 6 states We consider now the three-tangle for a family of rank-6 mixed states:

$$
\varrho(t)=t|G H Z, 2-\rangle\langle G H Z, 2-|+(1-t) \sigma,
$$

where

$$
\begin{aligned}
\sigma= & \frac{1}{11}|G H Z, 1+\rangle\left\langle G H Z, 1+\left|+\frac{1}{11}\right| G H Z, 1-\right\rangle\left\langle G H Z, 1-\left|+\frac{3}{11}\right| G H Z, 2+\right\rangle\langle G H Z, 2+| \\
& +\frac{3}{11}|G H Z, 3+\rangle\left\langle G H Z, 3+\left|+\frac{3}{11}\right| G H Z, 4+\right\rangle\langle G H Z, 4+| .
\end{aligned}
$$

From the analysis for our rank-5 states, we know that $\sigma$ has vanishing three-tangle, and the three-tangle for $\varrho(t)$ is given by

$$
\tau_{3}(\varrho(t))= \begin{cases}0, & 0 \leq t \leq t_{0} \\ g_{I}(t), & t_{0} \leq t \leq t_{1} \\ g_{I I}(t), & t_{1} \leq t \leq 1\end{cases}
$$

where

$$
\begin{aligned}
g_{I}(t) & =t^{2}+\frac{6}{11} t(1-t)-\frac{27-24 \sqrt{3}}{121}(1-t)^{2}-\frac{24 \sqrt{11}}{121} \sqrt{t(1-t)^{3}}, \\
g_{I I}(t) & =\frac{t-t_{1}}{1-t_{1}}+\frac{1-t}{1-t_{1}} g_{I}\left(t_{1}\right), \quad t_{0}=0.2143, \quad t_{1}=0.8290 .
\end{aligned}
$$


We have the optimal decomposition

$$
\varrho(t)= \begin{cases}\frac{t}{8 t_{0}} \sum \Pi_{i}\left(t_{0}\right)+\frac{t_{0}-t}{t_{0}} \sigma, & 0 \leq t \leq t_{0} \\ \frac{1}{8} \sum \Pi_{i}(t), & t_{0} \leq t \leq t_{1} \\ \frac{1-t}{8\left(1-t_{1}\right)} \sum \Pi_{i}\left(t_{1}\right)+\frac{t-t_{1}}{1-t_{1}}|G H Z, 2-\rangle\langle G H Z, 2-|, & t_{1} \leq t \leq 1\end{cases}
$$

where

$$
\begin{array}{ll}
\Pi_{1}(t)=|Z(t, 0,0,0,0,0)\rangle\langle Z(t, 0,0,0,0,0)|, & \Pi_{2}(t)=|Z(t, 0, \pi, \pi, 0,0)\rangle\langle Z(t, 0, \pi, \pi, 0,0)|, \\
\Pi_{3}(t)=|Z(t, \pi, 0, \pi, 0, \pi)\rangle\langle Z(t, \pi, 0, \pi, 0, \pi)|, & \Pi_{4}(t)=|Z(t, \pi, \pi, 0,0, \pi)\rangle\langle Z(t, \pi, \pi, 0,0, \pi)|, \\
\Pi_{5}(t)=|Z(t, \pi, 0, \pi, \pi, 0)\rangle\langle Z(t, \pi, 0, \pi, \pi, 0)|, & \Pi_{6}(t)=|Z(t, \pi, \pi, 0, \pi, 0)\rangle\langle Z(t, \pi, \pi, 0, \pi, 0)|, \\
\Pi_{7}(t)=|Z(t, 0,0,0, \pi, \pi)\rangle\langle Z(t, 0,0,0, \pi, \pi), & \Pi_{8}(t)=|Z(t, 0, \pi, \pi, \pi, \pi)\rangle\langle Z(t, 0, \pi, \pi, \pi, \pi)| .
\end{array}
$$

Obvious, all $\Pi_{i}\left(t_{0}\right)$ have vanishing three-tangle.

The case of rank-7 states The three tangle of the following rank-7 mixed states can be similarly calculated:

$$
\gamma(s)=s|G H Z, 3-\rangle\langle G H Z, 3-|+(1-s) \zeta,
$$

where

$$
\begin{aligned}
\zeta= & \frac{1}{34}|G H Z, 2-\rangle\left\langle G H Z, 2-\left|+\frac{3}{34}\right| G H Z, 1+\right\rangle\left\langle G H Z, 1+\left|+\frac{3}{34}\right| G H Z, 1-\right\rangle\langle G H Z, 1-| \\
& +\frac{9}{34}|G H Z, 2+\rangle\left\langle G H Z, 2+\left|+\frac{9}{34}\right| G H Z, 3+\right\rangle\left\langle G H Z, 3+\left|+\frac{9}{34}\right| G H Z, 4+\right\rangle\langle G H Z, 4+| .
\end{aligned}
$$

Applying the similar approach above we get

$$
\tau_{3}(\gamma(s))= \begin{cases}0, & 0 \leq s \leq s_{0} \\ g_{I}(s), & s_{0} \leq s \leq s_{1} \\ g_{I I}(s), & s_{1} \leq s \leq 1\end{cases}
$$

where

$$
\begin{aligned}
g_{I}(s) & =s^{2}+\frac{8}{17} s(1-s)-\frac{56-72 \sqrt{3}}{289}(1-s)^{2}-\frac{24 \sqrt{102}}{289} \sqrt{s(1-s)^{3}}, \\
g_{I I}(s) & =\frac{s-s_{1}}{1-s_{1}}+\frac{1-s}{1-s_{1}} g_{I}\left(s_{1}\right), \quad s_{0}=0.2062, \quad s_{1}=0.8375 .
\end{aligned}
$$

We also can get the corresponding optimal decomposition for $\gamma(s)$ :

$$
\gamma(s)= \begin{cases}\frac{s}{8 s_{0}} \sum \Pi_{i}\left(s_{0}\right)+\frac{s_{0}-s}{s_{0}} \zeta, & 0 \leq s \leq s_{0} \\ \frac{1}{8} \sum \Pi_{i}(s), & s_{0} \leq s \leq s_{1} \\ \frac{1-s}{8\left(1-s_{1}\right)} \sum \Pi_{i}\left(s_{1}\right)+\frac{s-s_{1}}{1-s_{1}}|G H Z, 3-\rangle\langle G H Z, 3-|, & s_{1} \leq s \leq 1\end{cases}
$$


where

$$
\begin{aligned}
& \Pi_{1}(s)=|Z(s, 0,0,0,0,0,0)\rangle\langle Z(s, 0,0,0,0,0,0)|, \\
& \Pi_{2}(s)=|Z(s, 0, \pi, 0, \pi, \pi, \pi)\rangle\langle Z(s, 0, \pi, 0, \pi, \pi, \pi)|, \\
& \Pi_{3}(s)=|Z(s, \pi, 0,0, \pi, 0, \pi)\rangle\langle Z(s, \pi, 0,0, \pi, 0, \pi)|, \\
& \Pi_{4}(s)=|Z(s, \pi, \pi, 0,0, \pi, 0)\rangle\langle Z(s, \pi, \pi, 0,0, \pi, 0)|, \\
& \Pi_{5}(s)=|Z(s, 0, \pi, \pi, 0,0, \pi)\rangle\langle Z(s, \pi, 0, \pi, \pi, 0,0, \pi)|, \\
& \Pi_{6}(s)=|Z(s, 0,0, \pi, \pi, \pi, 0)\rangle\langle Z(s, 0,0, \pi, \pi, \pi, 0)|, \\
& \Pi_{7}(s)=|Z(s, \pi, \pi, \pi, \pi, 0,0)\rangle\langle Z(s, \pi, \pi, \pi, \pi, 0,0)|, \\
& \Pi_{8}(s)=|Z(s, \pi, 0, \pi, 0, \pi, \pi)\rangle\langle Z(s, \pi, 0, \pi, 0, \pi, \pi)| .
\end{aligned}
$$

The case of rank- 8 states The rank of a three-qubit mixed state could be at most 8 . We now introduce a family of rank- 8 mixed states:

$$
\rho(r)=r|G H Z, 4-\rangle\langle G H Z, 4-|+(1-r) \eta,
$$

where

$$
\begin{aligned}
\eta= & \frac{1}{35}|G H Z, 3-\rangle\left\langle G H Z, 3-\left|+\frac{1}{35}\right| G H Z, 2-\right\rangle\left\langle G H Z, 2-\left|+\frac{3}{35}\right| G H Z, 1+\right\rangle\langle G H Z, 1+| \\
& +\frac{3}{35}|G H Z, 1-\rangle\left\langle G H Z, 1-\left|+\frac{9}{35}\right| G H Z, 2+\right\rangle\langle G H Z, 2+| \\
& +\frac{9}{35}|G H Z, 3+\rangle\left\langle G H Z, 3+\left|+\frac{9}{35}\right| G H Z, 4+\right\rangle\langle G H Z, 4+| .
\end{aligned}
$$

Obviously, $\tau_{3}(\eta)=0$. The three-tangle of $\rho(r)$ is given by:

$$
\tau_{3}(\rho(r))= \begin{cases}0, & 0 \leq r \leq r_{0} \\ g_{I}(r), & r_{0} \leq r \leq r_{1} \\ g_{I I}(r), & r_{1} \leq r \leq 1\end{cases}
$$

where

$$
\begin{aligned}
g_{I}(r) & =r^{2}+\frac{2}{5} r(1-r)-\frac{207-384 \sqrt{3}}{1225}(1-r)^{2}-\frac{128 \sqrt{105}}{1225} \sqrt{r(1-r)^{3}}, \\
g_{I I}(r) & =\frac{r-r_{1}}{1-r_{1}}+\frac{1-r}{1-r_{1}} g_{I}\left(r_{1}\right), \quad r_{0}=0.2490, r_{1}=0.8649 .
\end{aligned}
$$

The optimal decomposition for $\rho(r)$ can be similarly obtained:

$$
\rho(r)= \begin{cases}\frac{r}{8 r_{0}} \sum \Pi_{i}\left(r_{0}\right)+\frac{r_{0}-r}{r_{0}} \eta, & 0 \leq r \leq r_{0} \\ \frac{1}{8} \sum \Pi_{i}(r), & r_{0} \leq r \leq r_{1} \\ \frac{1-r}{8\left(1-r_{1}\right)} \sum \Pi_{i}\left(r_{1}\right)+\frac{r-r_{1}}{1-r_{1}}|G H Z, 4-\rangle\langle G H Z, 4-|, & r_{1} \leq r \leq 1\end{cases}
$$


where

$$
\begin{aligned}
& \Pi_{1}(r)=|Z(r, 0,0,0,0,0,0,0)\rangle\langle Z(P, 0,0,0,0,0,0,0)|, \\
& \Pi_{2}(r)=|Z(r, 0,0,0, \pi, \pi, \pi, \pi)\rangle\langle Z(r, 0,0,0, \pi, \pi, \pi, \pi)|, \\
& \Pi_{3}(r)=|Z(r, 0, \pi, \pi, 0,0, \pi, \pi)\rangle\langle Z(r, 0, \pi, \pi, 0,0, \pi, \pi)|, \\
& \Pi_{4}(r)=|Z(r, 0, \pi, \pi, \pi, \pi, 0,0)\rangle\langle Z(r, 0, \pi, \pi, \pi, \pi, 0,0)|, \\
& \Pi_{5}(r)=|Z(r, \pi, 0, \pi, 0, \pi, 0, \pi)\rangle\langle Z(r, \pi, 0, \pi, 0, \pi, 0, \pi)|, \\
& \Pi_{6}(r)=|Z(r, \pi, 0, \pi, \pi, 0, \pi, 0)\rangle\langle Z(r, \pi, 0, \pi, \pi, 0, \pi, 0)|, \\
& \Pi_{7}(r)=|Z(r, \pi, \pi, 0,0, \pi, \pi, 0)\rangle\langle Z(r, \pi, \pi, 0,0, \pi, \pi, 0), \\
& \Pi_{8}(r)=|Z(r, \pi, \pi, 0, \pi, 0,0, \pi)\rangle\langle Z(r, \pi, \pi, 0, \pi, 0,0, \pi)| .
\end{aligned}
$$

\section{CKW inequality}

Given a family of mixed three-qubit states with the corresponding three-tangle, one might check the CKW relations [12]. For a pure three-qubit state $|\psi\rangle \in \mathcal{C}^{2} \otimes \mathcal{C}^{2} \otimes \mathcal{C}^{2}$, with the reduced two-qubit density matrices $\rho_{A B}=\operatorname{Tr}_{C}(|\psi\rangle\langle\psi|), \rho_{A C}=\operatorname{Tr}_{B}(|\psi\rangle\langle\psi|)$ and $\rho_{A}=\operatorname{Tr}_{B C}(|\psi\rangle\langle\psi|)$, one has the monogamy relation $4 \operatorname{det}\left(\rho_{A}\right)=C\left(\rho_{A B}\right)^{2}+C\left(\rho_{A C}\right)^{2}+\tau_{3}(|\psi\rangle)$, where $C\left(\rho_{A B}\right)$ (resp. $\left.C\left(\rho_{A C}\right)\right)$ is the concurrence for the corresponding reduced state $\rho_{A B}$ (resp. $\left.\rho_{A C}\right), \tau_{3}(|\psi\rangle)$ is the three-tangle of $|\psi\rangle$. For mixed states, the following CKW inequality holds, $4 \min \left[\operatorname{det}\left(\rho_{A}\right)\right] \geq$ $C\left(\rho_{A B}\right)^{2}+C\left(\rho_{A C}\right)^{2}$. The CKW inequality has been examined for the mixture of GHZ and W states in [16] and the mixture of GHZ, W and flipped-W states in [18. In the following we check if the CKW inequality holds for the states introduced in our paper.

As an example, we consider the case of rank-5 states. It is direct to verify that these states satisfy $C\left(\rho_{A B}\right)^{2}+C\left(\rho_{A C}\right)^{2}=0$. And the minimum one-tangle is given by

$$
4 \min \left[\operatorname{det}\left(\rho_{A}\right)\right]=1-\frac{8}{5} p(1-p)-\frac{9}{25}(1-p)^{2}+\frac{6 \sqrt{30}}{25} \sqrt{p(1-p)^{3}} .
$$

From Fig. 2 we see that the CKW inequality is obviously satisfied. Moreover the inequality

$4 \min \left[\operatorname{det}\left(\rho_{A}\right)\right] \geq C\left(\rho_{A B}\right)^{2}+C\left(\rho_{A C}\right)^{2}+\tau_{3}(\psi)$ is also satisfied for these rank-5 states. In particular in the region $0 \leq p \leq p_{0}=0.7377$, both the concurrence and three-tangle are zero, but the onetangle is not zero.

\section{Conclusion}

We have constructed several classes of different ranked mixed states in three-qubit system. We have provided explicit expressions for the three-tangle and optimal decompositions for all these states. We have also studied the relations between the CKW inequality and these classes of 


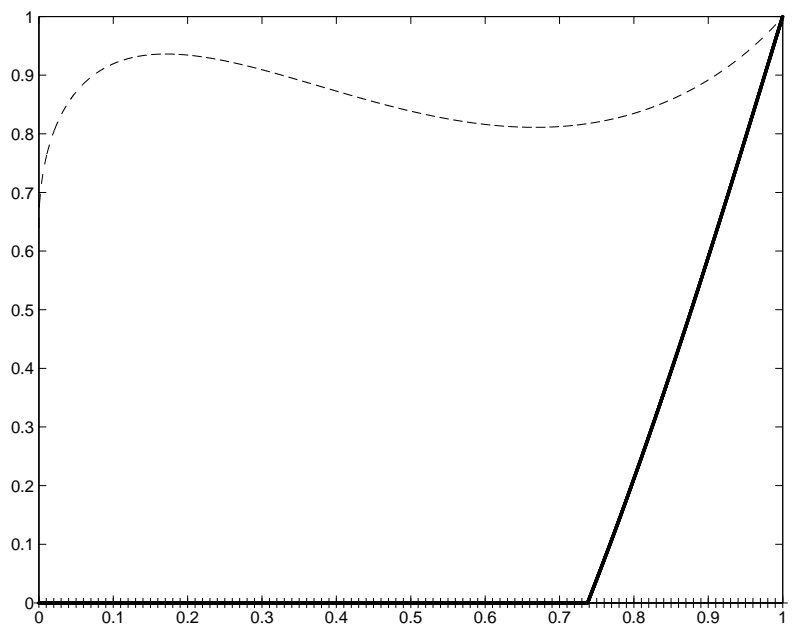

Figure 2: The $p$ dependence of one-tangle (upper dotted line), sum of squared concurrences (solid line along the horizontal axis) and three-tangle (solid line).

states, and shown that the CKW inequality are satisfied for these states. Concurrence of mixed two-qubit states has been applied to study quantum phase transitions. It has been shown that the pairwise entanglement of the nearest-neighbor two sites in spin- $1 / 2$ lattice models has special singularity at quantum critical points [22. It can be expected that multipartite entanglement would reveal further relations between the quantum phase transitions and quantum entanglement. Our results could help studies on applications of quantum entanglement in all these related researches.

Acknowledgments The work is supported by NSFC (Grant Nos.10871228, 10875081, 60873191, 60903152, 60821001), SRFDP (Grant No. 200800131016, 20090005110010), Beijing Nova Program (Grant No. 2008B51), Key Project of Chinese Ministry of Education (Grant No. 109014), and Beijing Municipal Education Commission No. KM200510028021 and No.KZ200810028013.

\section{References}

[1] M.A. Nilsen and I.L. Chuang, Quantum Computation and Quantum Information (2000).

[2] Ye Yeo and Wee Kang Chua, Phys. Rev. Lett. 96, 060502 (2006).

[3] N. Gisin and S.Massar, Phys. Rev. Lett. 79, 2153 (1997).

[4] A.Uhlmann, Phys. Rev. A 62, 032307 (2000); P. Rungta, V. Buzek, C.M. Caves, M. Hillery, and G.J. Milburn, Phys. Rev. A 64, 042315 (2001); S. Albeverio and S. M. Fei, J. Opt. B: Quantum Semiclass. Opt. 3, 223 (2001). 
[5] C.H. Bennett, D.P. DiVincenzo, J.A. Smolin, and W.K. Wootters, Phys. Rev. A 54, 3824 (1996).

[6] M. Horodecki, Quant. Inf. Comp. 1, 3 (2001); D. Bruß, J. Math. Phys. 43, 4237 (2002); K. Chen, S. Albeverio, and S.M. Fei, Phys. Rev. Lett. 95, 210501 (2005).

[7] C.H. Bennett, H.J. Bernstein, S. Popescu, and B. Schumacher, Phys. Rev. A. 53, 2046 (1996); B. Sutherland, Phys. Rev. Lett. 80, 2445 (1998); A. Uhlmann, Phys. Rev. A. 62, 032307 (2000); Y.C. Ou, H. Fan, and S.M. Fei, Phys. Rev. A. 78, 012311 (2008).

[8] Y.C. Ou and H. Fan, Phys. Rev. A. 75, 062308 (2007).

[9] X.H. Gao, S.M. Fei, and K. Wu, Phys. Rev. A. 74, 050303(R) (2006).

[10] X.H. Gao and S.M. Fei, Eur. Phys. J.Special Topics 159, 71 (2008).

[11] Y. Cao and A.M. Wang, J. Phys. A: Math. Theor. 40, 3507 (2007).

[12] V. Coffman, J. Kundu, and W.K. Wootters, Phys. Rev. A 61, 052306 (2000).

[13] A. Caley, 1845 Cambridge Math. J. 4, 193.

[14] A. Miyake, Phys. Rev. A 67, 012108 (2003).

[15] F. Benatti, A. Narnhofer, and A. Uhlmann, Rep.Math.Phys. 38, 123 (1996).

[16] R. Lohmayer, A. Osterloh, J. Siewert, and A. Uhlmann, Phys. Rev. Lett. 97, 260502 (2006).

[17] C. Eltschka, A. Osterloh, J. Siewert, and A. Uhlmann, New J.Phys. 10, 043014 (2008).

[18] E. Jung, M.R. Hwang, D.K. Park, and J.W. Son, Phys. Rev. A 79, 024306 (2009).

[19] E. Jung, D. Park, and J.W. Son, Phys. Rev. A 80, 010301(R) (2009).

[20] K. Cao, Z.W. Zhou, G.C. Guo, and L.X. He, arXiv: quant-ph 1001.0067.

[21] A. Osterloh, J. Siewert, and A. Uhlmann, Phys. Rev. A 77, 032310 (2008).

[22] L. Amico, R. Fazio, A. Osterloh, and V. Vedral, Rev. Mod. Phys. 80, 518 (2008). 